\section{Facharztprüfung zur Erlangung des Facharzttitels FMH für Neurologie}

Aufgrund des Weiterbildungsprogrammes, welches am 1. Januar 1996 in Kraft gesetzt wurde, ist die Teilnahme an der Facharztprüfung für diejenigen Kandidatinnen und Kandidaten Voraussetzung für die Erlangung des Facharzttitels FMH für Neurologie, welche ihre Weiterbildung in Neurologie am 31. Dezember 1996 nicht abgeschlossen hatten. Es empfiehlt sich, die Facharztprüfung frühestens im letzten Jahr der reglementarischen Weiterbildung abzulegen (Art. 23 WBO).
Ort: CHUV, Lausanne

Zeitpunkt: Freitag und Samstag, 29.-30. September 2000

Prüfungsgebühr: Die SNG erhebt eine Prüfungsgebühr von Fr. 500.-.

Anmeldung: Schriftliche Anmeldung unter Beilage des Ausbildungscurriculums an:

Prof. Dr. med. J. Bogousslavsky,

Service de Neurologie,

CHUV-BH 13, 1011 Lausanne,

Tel. 02131412 20, Fax 0213141231.

Anmeldefrist: 30. Juni 2000

\section{Examen de spécialiste en vue de l'obtention du titre FMH en neurologie}

Conformément au programme de formation postgraduée qui est entré en vigueur le $1^{\text {er }}$ janvier 1996, la participation à l'examen de spécialiste est obligatoire pour les candidats à l'obtention du titre FMH en neurologie qui n'avaient pas terminé leur formation postgraduée en neurologie le 31 décembre 1996. Il est recommandé de passer l'examen de spécialiste au plus tôt durant la dernière année de formation postgraduée réglementaire (art. 23 RFP).
Lieu: CHUV, Lausanne

Date: Vendredi et samedi, 29 et 30 septembre 2000

Taxe d'examen: La SSN prélève une participation aux frais de Fr. 500.-.

Inscription: A adresser par écrit en associant un curriculum de formation à l'adresse suivante:

Prof. J. Bogousslavsky,

Service de Neurologie,

CHUV-BH 13, 1011 Lausanne,

tél. 02131412 20, fax 0213141231.

Délai d'inscription: 30 juin 2000 